(C)2007 IEEE. Personal use of this material is permitted. However, permission to reprint/republish this material for advertising or promotional purposes or for creating new collective works for resale or redistribution to servers or lists, or to reuse any copyrighted component of this work in other works must be obtained from the IEEE 


\title{
Ontology-based Digital Ecosystem Conceptual Representation
}

\author{
Hai Dong, Farookh Khadeer Hussain, Elizabeth Chang \\ Digital Ecosystem and Business Intelligence Institute \\ Curtin University of Technology \\ Perth, Australia \\ Email: \{hai.dong, farookh.hussain, elizabeth.chang\}@cbs.curtin.edu.au
}

\begin{abstract}
Along with the evolution of Digital Ecosystem, in regards to its definition, two different theories have emerged, respectively from Soluta.net and from Digital Ecosystem and Business Intelligence Institute. This is paper explains the differences between the two theories and more importantly proposes a ontology-based conceptual representation for the concept of Digital Ecosystem with the purpose of helping researchers in the field to better understand the notion of Digital Ecosystem. In addition, we will introduce a simple ontology notation system, as the basis of our ontology model.
\end{abstract}

Keywords - Digital Ecosystem; ontology

\section{INTRODUCTION}

Digital Ecosystem is a neoteric terminology and its appearance is as a result of the natural existence of Business Ecosystem, along with the evolution of business network and information technology. The goal of digital ecosystems is to improve the efficiency of the communication between internal agents and to formally structure the existing Business Ecosystem. Since, the research on digital ecosystem is still in its infancy, interpretations of some basic concepts regarding Digital Ecosystems are still debated. The definition of digital ecosystem is one of them, which is defined in two distinct ways. Soluta.net define Digital Ecosystems from structural and functional perspective, which perceives digital ecosystem as an open-source network environment for carrying out business activities, mainly intended towards or focusing on small and medium enterprises[3]. Another alternative definition of Digital Ecosystems is provided by the Digital Ecosystem and Business Intelligence Institute (DEBII) from the perspective of specialization, which sees it as "an open, loosely coupled, domain clustered, demand-driven, self-organizing and agentbased environment which each species is proactive and responsive for its own benefit and profit" [2] [4]. The former definition and related theories focus on the introduction and implementation of the digital ecosystem architecture from a pure technical perspective. By constructing the service-oriented environment by means of network technology, BML (Business Modeling Languages), SDL (Service Description Languages), ontology etc, digital ecosystem realizes the function of service creation, service execution and service optimization [3]. The latter definition concentrate on defining the existing concepts within Digital Ecosystems, network architecture and swarm intelligence in digital ecosystem, which tends to conceptualize and formally structure its components. Thus, the former definition is focused towards the actual implementation and commercialization, while the latter definition is more inclined towards conceptualization.

To solve the resulting conceptual ambiguity and to help researchers to better understand what digital ecosystem is, we employ ontology which is seen as an affective tool to assist people share knowledge. We intend to develop a conceptual model of digital ecosystem. Since the objective of this paper is to conceptualize the components of digital ecosystem, the theory/definition on which our research is the second definition we discussed above.

\section{NOTATION SYSTEM FOR ONTOLOGY REPRESENTATION}

Before we represent the ontological model for digital ecosystem, a notation system for representing ontology is introduced in this section. The notation system utilized in the ontological representation is based on Chang et al.'s work, which consists of four basic notations as Table 1[1]. Although in the past we usually used to employ UML (Unified Modeling Language) to represent an ontology model, due to its complex symbols categories, UML cannot efficiently help people better understand the shared knowledge. This notation system simplifies the symbols and its symbols are closer to the principle of ontology which is the combination of shared concepts and relationships between concepts [5].

TABLE I. ONTOLOGY NOTATION SYSTEM

\begin{tabular}{|c|c|}
\hline Ontology Notation & Semantics of the Notation \\
\hline & $\begin{array}{l}\text { Double-field Box represents the Ontological } \\
\text { Concepts. }\end{array}$ \\
\hline relation & $\begin{array}{l}\text { A dotted line represents Ontology Concept } \\
\text { Association Relation which represents a } \\
\text { Concept is closely related to another concept. } \\
\text { The relationship name can be noted above the } \\
\text { dotted line. }\end{array}$ \\
\hline & $\begin{array}{l}\text { Open-arrow line represents Composition and } \\
\text { Aggregation or Part-of relationship between } \\
\text { Upper Ontology Concept and Lower Ontology } \\
\text { Concept. }\end{array}$ \\
\hline & $\begin{array}{l}\text { Solid-arrow line represents Generalization and } \\
\text { Specification relation, which is a relation } \\
\text { between Upper Generic Concept and Lower } \\
\text { Specific Concept. }\end{array}$ \\
\hline & $\begin{array}{l}\text { Circle-line represents the instance of } \\
\text { Ontological Concepts. }\end{array}$ \\
\hline
\end{tabular}




\section{Digital Ecosystem Ontology}

\section{A. Hierarchy of Digital Ecosystem Ontology Concepts}

Broadly speaking, digital ecosystem consists of two basic parts or components - species and an ecosystem environment. Each species can be viewed as an individual or an organization and has its own role to play. The species work together to take care of their living environment; on the other hand, environment supports species' survival and development. There are three basic types of species - biological species, economic species and digital species. Thus, the hierarchy of digital ecosystem concepts is shown in Figure 1, below using the above introduced notation system.

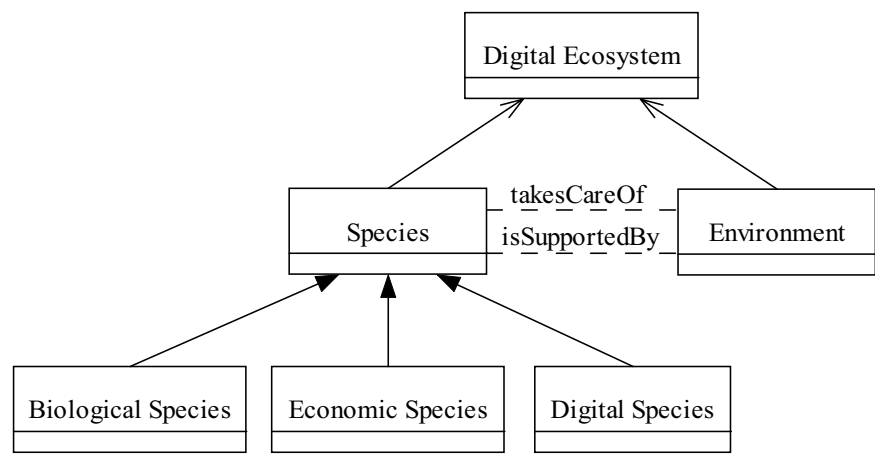

Figure 1. Digital ecosystem concepts hierarchy.

\section{B. Species Ontology}

Species is the basic element of digital ecosystem, which can be defined as the conceptualization of species which interacts with other species, shares commonly agreed vocabulary such as Ontology, comes from certain domain, plays dual roles (role of a server in which it provides available services to other species and role of a client, who request service), follows the rules (norms or regulations) of digital ecosystem, is proactive for or is responsive for own profit or benefit and carries out tasks that relates to Profit or Benefit.

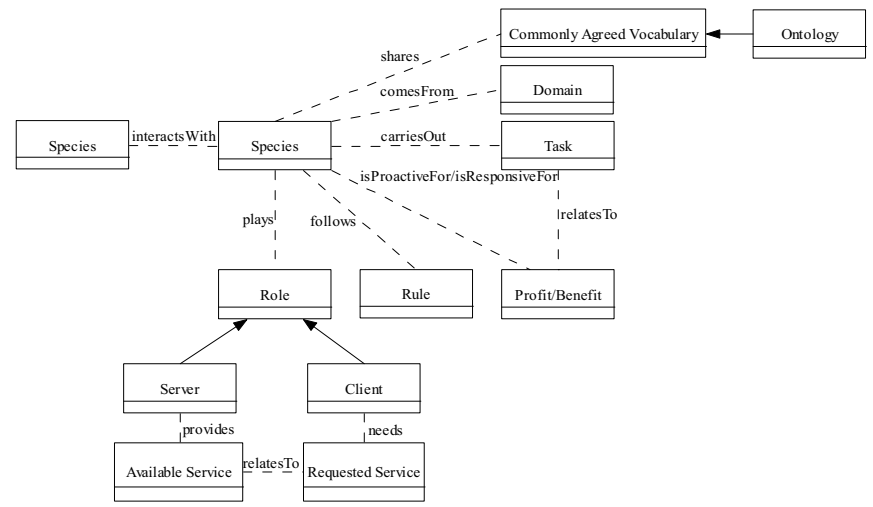

Figure 2. Species Ontology.

We present the Species Ontology as the combination of ontology name and a tuple where the elements of the tuple can be complex elements as defined below:
Species [Species, Commonly Agreed Vocabulary, Ontology, Domain, Task, Profit/Benefit, Rule, Role, Server, Client, Available Service and Requested Service] where:

'Species' refers to the definitions above.

'Commonly Agreed Vocabulary' refers to the common terms used by species to communicate with each other [4].

'Ontology' is a conceptualization of knowledge in a special domain [5].

'Domain' refers to a field in digital ecosystem where a species has common interest with other species [4].

'Task' refers to a mission that a species is executing, to survive and achieve its goals in the environment of digital ecosystem.

'Profit' or 'Benefit' means a social or economical advantage that a species gains by digital ecosystem [4].

'Rule' refers to a common or special regulation that a species should follow in the environment of digital ecosystem.

'Role' refers to the role of a specie in the interaction with other specie(s). There are two major roles in digital ecosystem interactions - server and client. In contrast to the other network architectures, in which each agent has a fixed role, specie(s) in digital ecosystem are able to play the dual roles at the same time [4].

'Server' is a role of a species that provides a service [4].

'Client' is a role of a service that requests a service [4].

'Available Service' is a service that a supplier provides [4].

'Requested Service' is a service that a requester needs [4].

\section{Biological Species Ontology, Economic Species Ontology and Digital Species Ontology}

Broadly speaking there are three subclasses of species, namely biological species, economic species and digital species. These subclasses of species inherit all the properties from the species concept. The major differences between them are their living environment, commutative object and rule.

An example of biological specie is the human specie. They live in the environment of natural ecosystem and interact with other humans. As a member of the society, they must follow the common rules in the human society (such as law, regulations etc). 


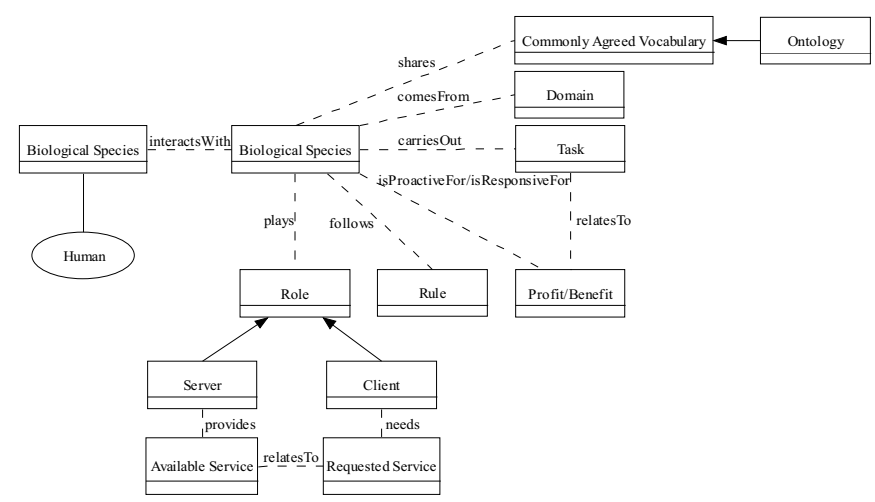

Figure 3. Biological Species Ontology.

Organization is one example of Economic Species. Organizations exist in the business environment and their commutative scope is limited in themselves. Organization is the aggregation of individuals. Since organizations belong to certain industries, they must follow some domain-specific rules (such as industrial agreements).

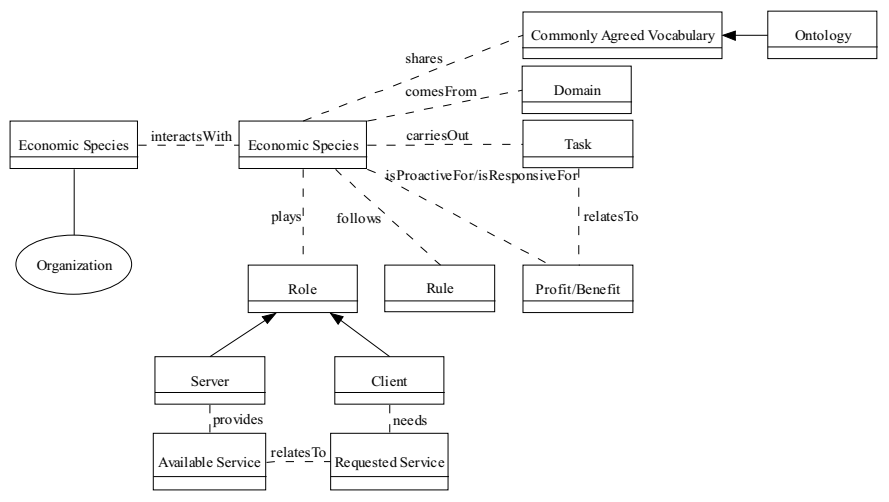

Figure 4. Economic Species Ontology.

Digital Species mainly includes hardware and software. Digital species are connected by networks and they also communicate with each other via networks, thus their living environment is digital network. The rules that may exist in the digital network world are protocols etc.

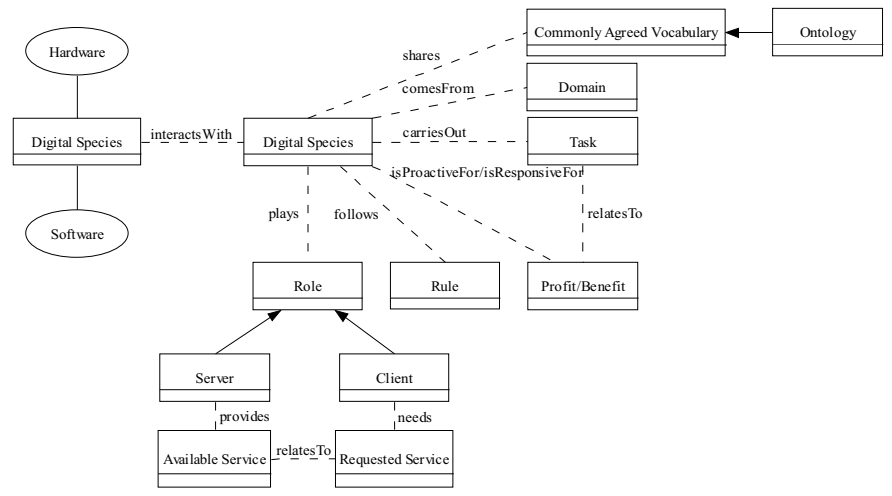

Figure 5. Digital Species Ontology.

\section{Environment Ontology}

In context of digital ecosystem, the environment can be defined as the conceptualization of Environment that is supported by Technologies and has the specifications of Open, Loosely Coupled, Demand-driven, Domain-Clustered, Selforganizing, and Agent-based. The Environment Ontology is shown in Fig. 6 through the use of notation system.

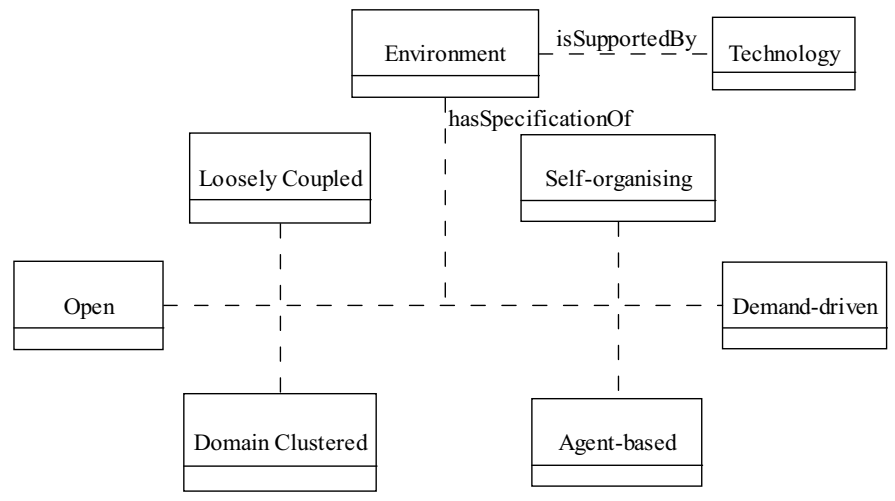

Figure 6. Environment Ontology.

We present the Environment Ontology as the combination of ontology name and a tuple where the elements of the tuple can be complex elements as defined below:

Environment [Environment, Technology, Open, Loosely Coupled, Demand-driven, Domain Clustered, Self-organizing and Agent-based] where

'Environment' refers to definition above.

'Technology' will be defined in the next section.

'Open' refers to a transparent environment where all interactions are visible [4].

'Loosely Coupled' refers to a freely bound and open relationship between species in digital ecosystem [4].

'Demand-driven' means that species actively join a community on their own interest [4].

'Domain Clustered' refers to an environment which consists of the field where some species have common interest [4].

'Self-organizing' means that species are capable of acting autonomously, making decisions and carrying out tasks in digital ecosystem [4].

'Agent-based' refers to an environment which contains human individuals, information technologies and tools that facilitate interaction and knowledge sharing between agents along with the resources that help maintain synergy between he agents [4].

\section{E. Technology Ontology}

We classify the technologies that support Digital Ecosystems into three main categories - extended web architecture, ontology-based knowledge sharing and a swarm intelligence-based self-organizing recommendation system. The concept of extended web architecture will be discussed in next section. Ontology-based knowledge sharing is to use ontological concepts as the major means to share multidisciplinary knowledge between different species. Swarm intelligence-based recommendation system is a platform on 
which species utilize swarm intelligence to cooperate and communicate with each other [4]. The ontology of swarm is defined in the following section.

The hierarchy of the concept of Technology is shown in Figure 7, through the use of notation system.

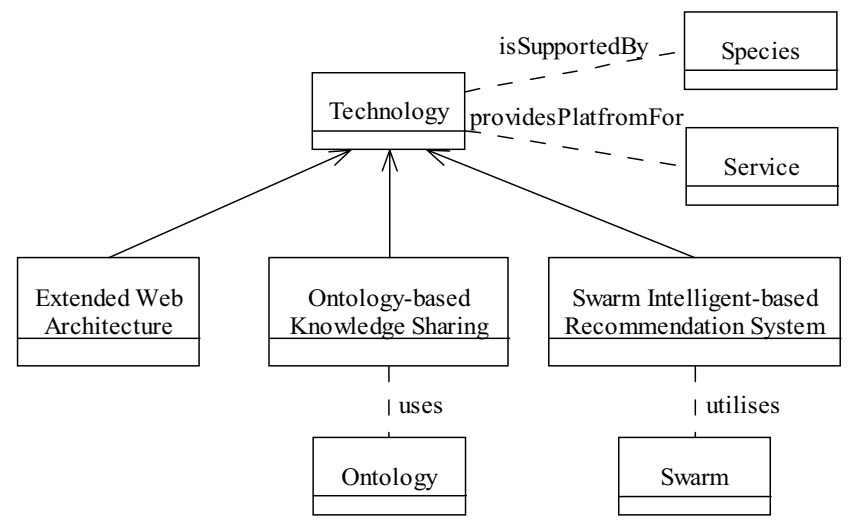

Figure 7. Technology Ontology.

\section{F. Extended Web Architecture Ontology and Its \\ Subcomponent Ontologies}

Extend web architecture of digital ecosystem contains five basic subclass architectures which are client-server architecture, peer-to-peer architecture, grid architecture, mobile and ad-hoc architecture and web-service architecture. Digital ecosystem architecture is constructed by the optional combination of one or more architectures. The detailed infrastructure of digital ecosystem architecture is based on the definition proposed by Soluta.net (www.digitalecosystem.org). What we study in this paper is the conceptual model of digital ecosystem architecture which is shown in Figure 8 .

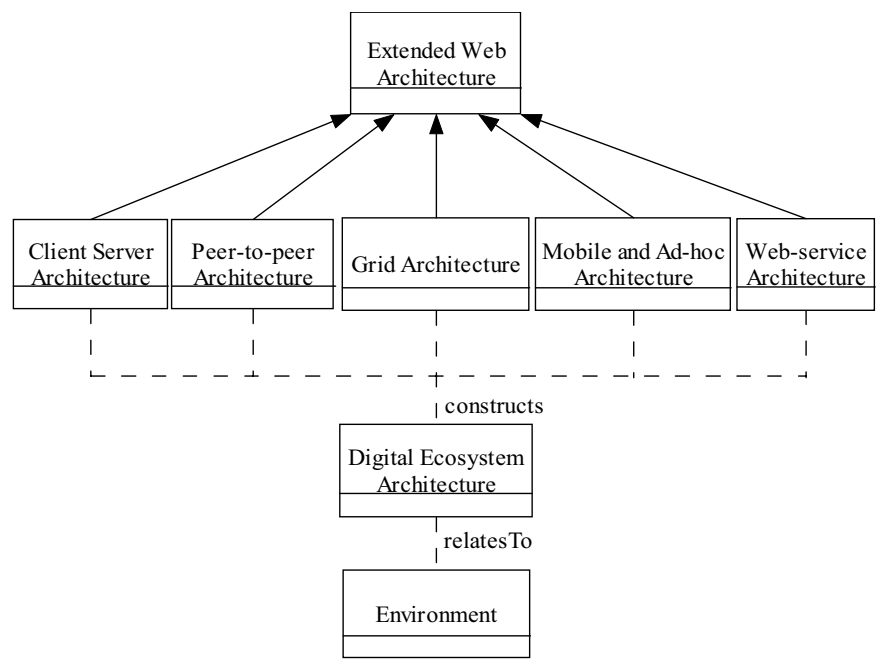

Figure 8. Extended Web Architecture Ontology.

In this section, we will define the ontological models for the five basic subcomponents of extended web architecture in digital ecosystem.

In client server architecture, a server is centered and fully controls the communication between surrounded clients; on the other hand, the function of each client also depends on the function of server. The role of client and server is fixed and canot be changed [4].

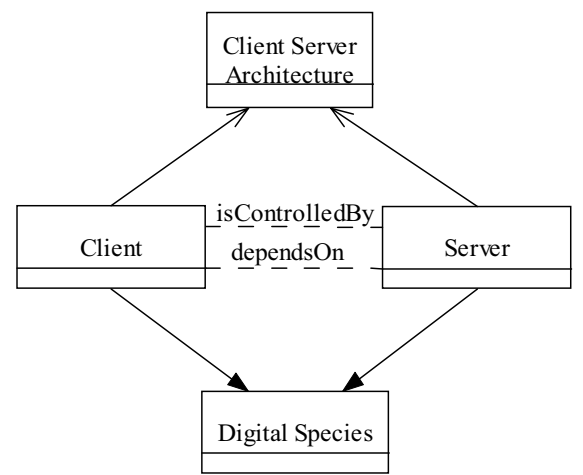

Figure 9. Client Server Architecture Ontology.

Peer-to-peer network consists of distributed peers which has same role and functions. There is no centralized server in this network. The communicating ability and computing powers of peer-to-peer network relies on the peers at the end of each connection [6].

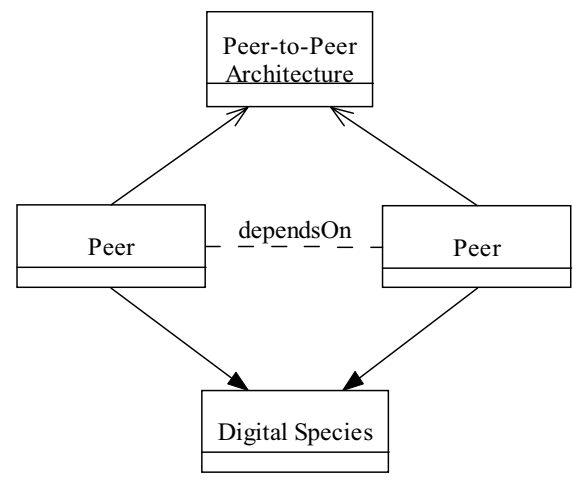

Figure 10. Peer-to-Peer Architecture Ontology.

Grid architecture aggregates geographically dispersed and autonomous resources and runs dynamically based on the capability of each node in a grid [7]. The difference between $\mathrm{P} 2 \mathrm{P}$ and grid is that in the former, there is a centralized resource manager which manages all the peers which work cooperatively while in the latter, there are distributed resource managers for each node, which support the integration of heterogeneous systems [4].

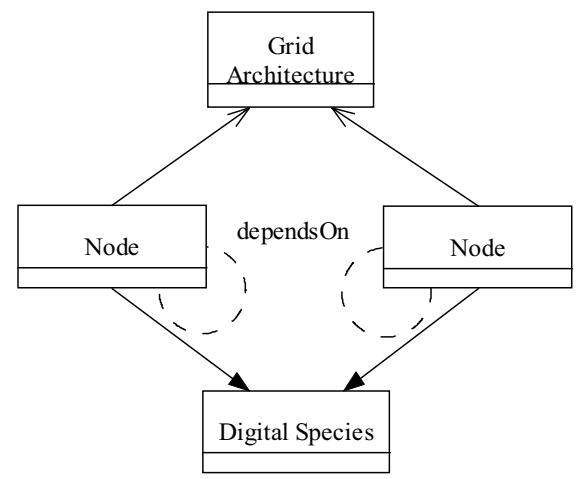


Figure 11. Grid Architecture Ontology.

Mobile and ad-hoc network have a great of similarity between them, with the major similarities being that the connection between the nodes is flexible; the main components of the network include compact devices and a server. The centralized server is fixed and controls other devices in the period of connection. In mobile network, the geographical location of compact devices is flexible. In ad-hoc network, the connection between compact devices and server is temporary. The compact devices' communication and other functions depend on the server's function.

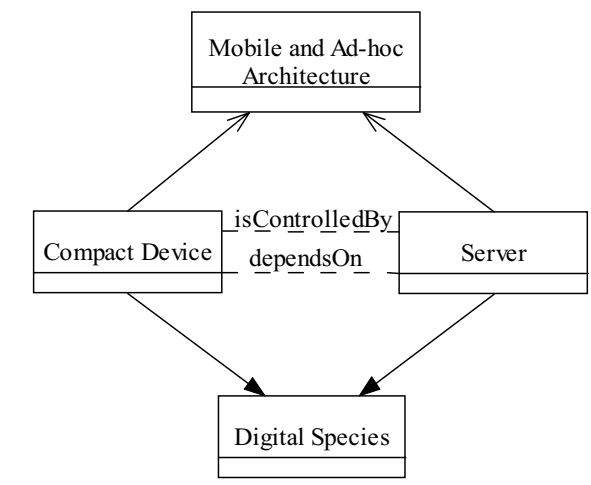

Figure 12. Mobile and Ad-hoc Architecture Ontology.

Web-service architecture involves three basic components service requester, service broker and service provider. A service provider is to publish an available service to a service broker. A service requester is to request a service from a service broker. The mission of a service broker is to find and bind a proper service requester for a service provider, or a proper service provider for a service requester [8]. A service broker is centralized, and service requesters and service providers are distributed [4].

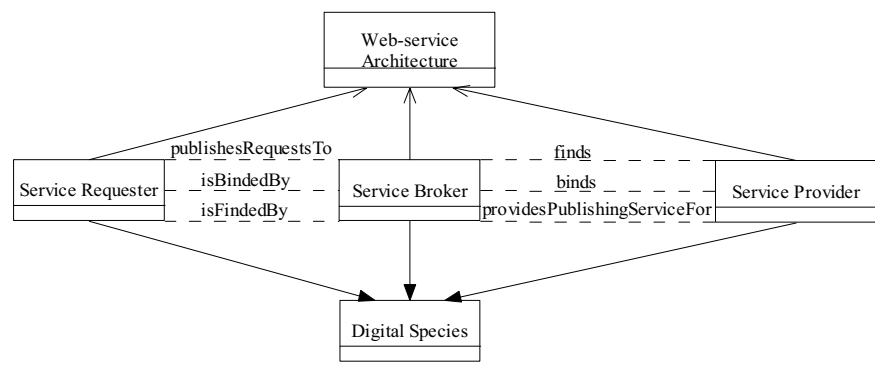

Figure 13. Web-service Architecture Ontology.

\section{G. Swarm Ontology and Leading Species Ontology}

In digital ecosystem, as we discussed in the prior section, due to the specification of self-organizing and collaboration, species may form an organization with flexible structures to cooperate and communicate. Here a concept - swarm, which is basically a set of species which has common characteristic and is able to interact and engage directly or indirectly with each other comes into picture. With the swarm intelligence technology, species that have common interest may group and cooperate, to share a problem and to collaboratively carry out a task. The structure of swarm may vary from time to time, which depends on its mission. For example, a hierarchical swarm is convenient for organizational management and the definition of communication channels; a linear swarm is beneficial for species to efficiently execute sequential tasks or flow operations; a circular swarm is useful for species to cooperate and comprehensively tackle issues.

In a swarm, a leader is chosen from species, to direct and facilitate the swarm, to represent the swarm to interact with other swarms [4]. Besides these functions, the leading species has same features as other species.

The ontological representation of Swarm Ontology and Leading Species Ontology is shown in Fig. 14 and Fig. 15.

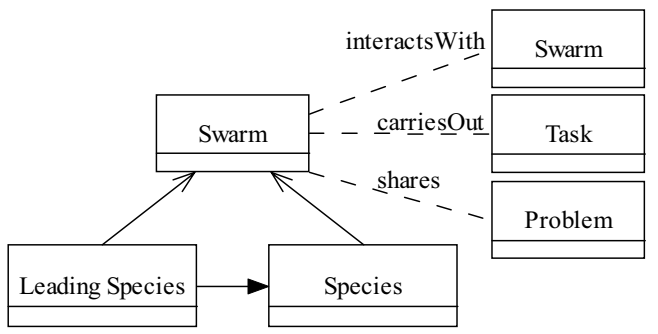

Figure 14. Swarm Ontology.

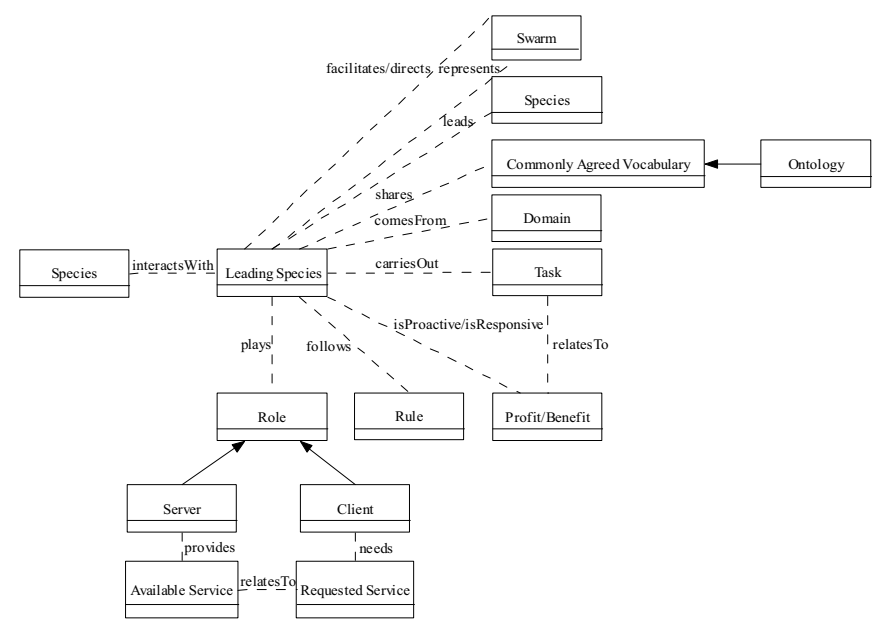

Figure 15. Leading Species Ontology.

\section{CONCLUSION AND FUTURE WORKS}

In this paper, we use ontology to represent the conceptual model of digital ecosystem and its subcomponents, based on the theory presented by Chang and West. The outcome we achieved in this paper is concluded below:

This paper proposes and explains the concept of digital ecosystem and its subclass concepts - Species and Environment. In addition, the specifications of Species Natural Species, Economic Species and Digital Species are defined through use of a special notation system. Furthermore, we define the concept about Technology and its subcomponents that support the digital ecosystem environment, including the five categories of extended web architectures which construct the mainframe of digital ecosystem architecture, and the swarm intelligence as the basic organizational style in digital ecosystem. 
The limitations in our research are as below:

This paper chooses only one digital ecosystem theory (DEBII) as its research goal, which supports limited knowledge to researchers.

The ontological representation towards services provided by digital ecosystem is brief and surface, which needs more detailed and deeper research in the future.

Our conceptual model has not been realized and validated in the real world.

Thus, against the above drawbacks in our research, in the future, we will extend our ontology design to deeper substructure and combine the theory of Soluta.net to our prototype, in order to create a unified and widely recognized conceptual model. Moreover, we will explore the function of digital ecosystem towards species by case study and literal research. Finally, we will prototype the ontological model in Protégé-owl and validate it in the real world by collecting other researchers' responses.

\section{REFERENCES}

[1] E. Chang, T. S. Dillon and F.K. Hussain, Trust and Reputation for Service Oriented Environments-Technologies for Building Business Intelligence and Consumer Confidence, London: John Wiley \& Sons, 2005, pp.131-153.

[2] E. Chang, M. Quaddus and R. Ramaseshan, "The vision of DEBI Institute: digital ecosystems and business intelligence," DEBII, 2006.

[3] P. Ferronato, "Introduction to the digital business ecosystem project," presented at MDA Technical Forum, Tokyo, 2004.

[4] E. Chang and M. West, "Digital Ecosystem - A next generation of the collaborative environment," presented at iiWAS 2006, Yogyakarta, 2006.

[5] N. Guarino, "Ontology-driven conceptual modeling," presented at $21 \mathrm{st}$ International Conference on Conceptual Modelling, Tampere, 2002, p.10.

[6] D. C. Verma, Legitimate Applications of Peer-to-Peer Networks, John Wiley \& Sons, Inc., 2004, pp. 1-17.

[7] "Grid Computing Info Centre," vol. 2007, R. Buyya, Ed.: GRID Infoware.

[8] "Web Services Architecture," vol. 2007, D. Booth, H. Haas, F. McCabe, E. Newcomer, M. Champion, C. Ferris, and D. Orchard, Eds.: W3C, 2004. 